\title{
Effect of Doxorubicin on Histomorphology of Liver of Wistar Albino Rats
}

Deepak Chaudhary ${ }^{1}$, Saroj Khatiwada ${ }^{2}$, Surendra Kumar Sah ${ }^{1}$, Man Kumar Tamang ${ }^{3}$, Soumya Bhattacharya ${ }^{4}$ and Chandra Bhushan Jha ${ }^{4}$

1. M.Sc. Human Anatomy, Department of Anatomy, Nobel Medical College, Biratnagar, Nepal

2. M.Sc. Medical Biochemistry, Department of Biochemistry, Modern Technical College, Lalitpur, Nepal

3. M.Sc. Medical Biochemistry, Department of Nutrition and Dietetics, Central Campus of Technology, Dharan, Nepal

4. MD Anatomy, Department of Human Anatomy, B P Koirala Institute of Health Sciences, Dharan, Nepal

\begin{abstract}
Doxorubicin is associated with adverse effects on organs like liver. This study was done to find the effects of doxorubicin on liver of wistar albino rats. Sixty healthy wistar albino rats were taken for the study. The rats were randomly divided in to 4 groups $(2$ experimental groups; group A1 and A2, and 2 control groups;group B1and B2 each containing 16 rats). The experimental groups were given a single dose of doxorubicin i.e. $10 \mathrm{mg} / \mathrm{kg}$ body weight intraperitoneally and sacrificed after 7 days and 14 days for each group. Rats under control groups were given a single intraperitoneal dose of $2.5 \mathrm{ml} / \mathrm{kg}$ body weight normal saline and sacrificed with their respective experimental groups. A significant difference $(p<0.001)$ and $(p=0.005)$ in final body weight was observed among group A1 experimental and B1 control rats, and among group A2 experimental and B2 control rats. As compared to group B2 control, group A2 experimental rats had significantly $(p=0.043)$ lower liver weight. Diameter of hepatocyte $(p<0.001$ for both groups) and nucleus $(\mathrm{p}=0.004$ for group A1 and control B1, and $\mathrm{p}<0.001$ for group A2 and control B2) was significantly higher in experimental rats as compared to their respective controls. Cross-sections of the liver of both control groups had normal architecture. However, there was progressive destruction of liver architecture across the experimental groups. Doxorubicin causes the disruption of normal architecture of liver in rats. Thus care needs to be taken during doxorubicin chemotherapy to minimize effects on liver.
\end{abstract}

Key words: Doxorubicin, Histomorphometry, Liver, Wistar albino rat.

\section{Introduction}

The anthracycline antibiotic, doxorubicin, is one of the most effective anti-tumour agent used to treat human malignancies [1]. It strives to kill malignant cells and produces tumor regression in a variety of human neoplasms. However, its clinical usefulness is limited by its proven toxicity to certain organs particularly the heart and the kidney [2]. Its dose dependent cardiotoxicity is the most serious side effect causing withdrawal of drug from hard chemotherapeutic regimen [3]. That's why its toxicity limits the usage in cancer chemotherapy [4].

Doxorubicin can kill both healthy and cancerous cells; however it has a greater effect on cells that are

Corresponding author:Saroj Khatiwada, Department of Biochemistry, Modern Technical College, Lalitpur, Nepal. multiplying rapidly [2]. Doxorubicin is metabolized predominantly by the liver to the major metabolite, doxorubicinol, and several cytotoxic aglycone metabolites [5, 6]. To undergo hepatic metabolism, drugs must first cross the hepatic sinusoidal endothelium to enter the space of disse and gain access to the hepatocytes [7].

Long term treatment with doxorubicin is limited by its hepatotoxicity [7]. A major limitation to its effect is the development of multi drug resistance of cancer cells [8]. Histopathological changes such as necrosis, hepatocyte degeneration, sinusoidal dilatation, haemorrhage and vascular congestion and dilatation also occur [9]. Because of numerous metabolic pathways, the liver is especially exposed to the harmful effect of exogenous substances, including drugs [10]. 
Considering the possible detrimental effect of doxorubicin on liver, we sought to find out the effect of doxorubicin on histomorphological characteristics of liver of wistar albino rats over short and long term as compared to controls.

\section{Materials and Methods}

A study was done among 60 healthy albino rats weighing 150-200gm at the department of anatomy of B P Koirala Institute of Health Sciences, Dharan, Nepal in 2013 to find the effects of doxorubicin on histomorphology of rat liver. The rats were randomly divided in to 2 groups (group A: experimental, and group B: control) each containing 32 rats. Group A was again divided into two sub-groups $\mathrm{A} 1$ and $\mathrm{A} 2$. Experimental rats were administered a single dose of $10 \mathrm{mg} / \mathrm{kg}$ body weight of doxorubicin intraperitoneally, and sacrificed after 7 days and 14 days for group A1 and A2 respectively. Control animals (group B1 and B2) received a similar volume of $0.9 \%$ saline intraperitoneally and were sacrificed at 7 and 14 days respectively. Rats were housed in well ventilated room at controlled ambient temperature $(25 \pm 5 \mathrm{C})$ with a 12 hours alternating light-dark cycle and fed standard rodent diet and water. The study protocol was approved by institute review boardof B P Koirala Institute of Health Sciences, Dharan, Nepal. All the experimental works were carried out as per the ethical guidelines of NHRC (Nepal Health Research Council) for the care and use of animals in health research in Nepal.

The weight of all experimental and control animals were taken initially and the final weights at the time of sacrifices were noted. The animals were anesthetized with ether soaked in cotton and their livers were fixed by in vivo perfusion technique. They were kept on the dissecting tray with their ventral surface facing upward and all four limbs pinned. The abdomen was opened to expose the abdominal aorta and inferior venacava. An 18 gauge needle was inserted on the abdominal aorta and tied with thread to keep it in constant position. The needle was attached to clean flask tube, connected with two bottle containing bouin's fluid and physiological saline separately. Then the chest was opened and the right atrium was nicked by a scissor to permit the drainage of blood. Thereafter, the physiological saline was perfused to flush out the blood. The fixative bouin's fluid was then transfused with the help of three ways stopcock. Approximately $200 \mathrm{ml}$ of each fluid was perfused. Perfusion was stopped when clear fixative drops start oozing out from snout of the animal. After completion of perfusion the liver were isolated from the abdomen with the help of scalpel and forceps and post fixed for 24 hours by the same fixative. The weights of the liver were measured by electronic balance. Then, the livers were cut into small pieces about the size of $5 \mathrm{~mm}$ to fix in bouin's fluid for 7-8 days. After 7-8 days of fixation in bouin's fluid, the tissue was processed and histological slides were prepared. Diameter of each hepatocyte was measured by two directions and mean of two was taken as a diameter of that hepatocyte using micrometer.

The data from the study were entered in the microsoft excel software program. Statistical analysis was performed using the SPSS (Statistical Package for Social Sciences) 19 version. The data were expressed as mean \pm SD. Student's t-test was used to determine the statistically significant difference in histomorphological cells, body and liver weight and sizes of cells between experimental and control at 95\% confidence interval. A p value less than 0.05 was considered statistically significant.

\section{Results}

The study comprised 64 wistar albino rats (32 experimental and 32 controls). It was observed that rats of experimental group A1 showed the loss of appetite and occurrence of diarrhoea. There were decreased locomotory activities. Bleeding from the nose was also seen. In group A2 experimental rats, appetite was seen to increase. Controls rate of both group showed healthy behavior being playful and having better food consumption. The mean initial body weight, final body 
weight, liver weight, hepatocyte diameter and nucleus diameter is shown in Table 1. A significant difference in final body weight was observed among group A1 experimental and $\mathrm{B} 1$ control rats, and among group $\mathrm{A} 2$ experimental and B2 control rats. As compared to group B2 control, group A2 experimental rats had significantly lower liver weight. Diameter of hepatocyte and nucleus was significantly higher in experimental rats as compared to their respective controls.

Cross-section of liver of control, group A1 and A2 rats is shown in Fig. 1. In group A1 experimental rats, lobes of the liver were seen to be adhered to each other in most of the animals and necrosis was seen. The liver section showed the histological architecture disrupted to some extent. There was degeneration of hepatic cords. The vacuolated cells with round to oval and elliptical shaped nuclei were seen. The sinusoidal space was also seen to be dilated to some extent. Some pathological changes such as necrosis, irregular cell membrane, irregular clumping of chromatin like mass were also seen. There was increase in the size of the hepatocytes and the nuclei. In group A2 experimental rats, livers of some of the animals showed lobes adhered to each other. The histological features showed some of the cells to be vacuolated with round to oval shaped nuclei. Dilated hepatic sinusoids were also seen with necrotic areas. In both the control group rats, liver morphology was normal and liver section displayed normal histological architecture. The hepatic cords were seen to be normal radiating from the central canal towards the periphery of the hepatic lobule with patent sinusoidal space between them. Sizes of the hepatocytes were normal, polygonal shaped having round to oval shaped prominent nuclei.

\section{Discussion}

In the present study we investigated the changes in histomorphology of liver in rats given doxorubicin after 1 and 2 weeks as compared to control. The rats of experimental group demonstrated loss of appetite and weight, diarrhea, redness around the nose and mouth, and yellowish coloration. Similar features including loss of hairs in certain areas of the body were observed by Osama et al. in the animals that received doxorubicin [3]. The weight loss observed in experimental rats might be due to loss of appetite causing the shedding of fat, and changes in the metabolic rates.

In the present study, decrease in weight of the liver of the experimental group was seen though not statistically significant. Similarly, rats given doxorubicin showed significantly higher hepatocyte diameter than controls. We observed increase in diameter of nucleus of hepatocytes in rats treated with doxorubicin. In the study by the Osama et al. the electron microscopic ultrastructure of hepatocytes of rats (DOX 1 week) showed changes in the nucleus shape, outer and inner membrane fused together and oval in shape. Condensed chromatin masses and appearance of vacuolization in the cytoplasm were also seen and at 2 weeks the hepatocytes injury was more severe [3]. In a study by Kelvin et al. drug-eluting microspheres/beads loaded with doxorubicin delivered to rats showed tumor necrosis minimal 1 hour after treatment and greatest at 7 days after the therapy [11].

Table 1 Anatomical and histomorphometric parameters in wistar albino rats.

\begin{tabular}{lllllll}
\hline Parameters & Group A1, $\mathrm{n}=16$ & Group B1, $\mathrm{n}=16$ & $\mathrm{P}$ value & \multicolumn{4}{l}{ Group A2, $\mathrm{n}=16$ Group B2, $\mathrm{n}=16$ P value } \\
\hline Initial body weight & $157.86 \pm 4.68$ & $155.31 \pm 5.31$ & 0.178 & $155.94 \pm 4.90$ & $156.25 \pm 5.00$ & 0.860 \\
Final body weight & $138.93 \pm 11.79$ & $159.06 \pm 5.54$ & $<0.001$ & $146.54 \pm 17.48$ & $163.44 \pm 5.69$ & 0.005 \\
Liver weight & $6.33 \pm 0.60$ & $6.33 \pm 1.15$ & 0.991 & $6.29 \pm 0.95$ & $6.90 \pm 0.26$ & 0.043 \\
Diameter of hepatocyte & $18.37 \pm 1.75$ & $15.41 \pm 1.32$ & $<0.001$ & $17.16 \pm 1.34$ & $15.17 \pm 1.60$ & 0.001 \\
Diameter of nucleus & $7.92 \pm 1.11$ & $6.89 \pm 0.65$ & 0.004 & $7.83 \pm 0.86$ & $6.62 \pm 0.757$ & $<0.001$ \\
\hline
\end{tabular}

The data is expressed as mean \pm SD. P value was calculated at $95 \%$ confidence interval. Group A1 and A2 received doxorubicin and were sacrificed at 7 and 14 days respectively. Control rats (group B1 and B2) were sacrificed at 7 and 14 days respectively. 

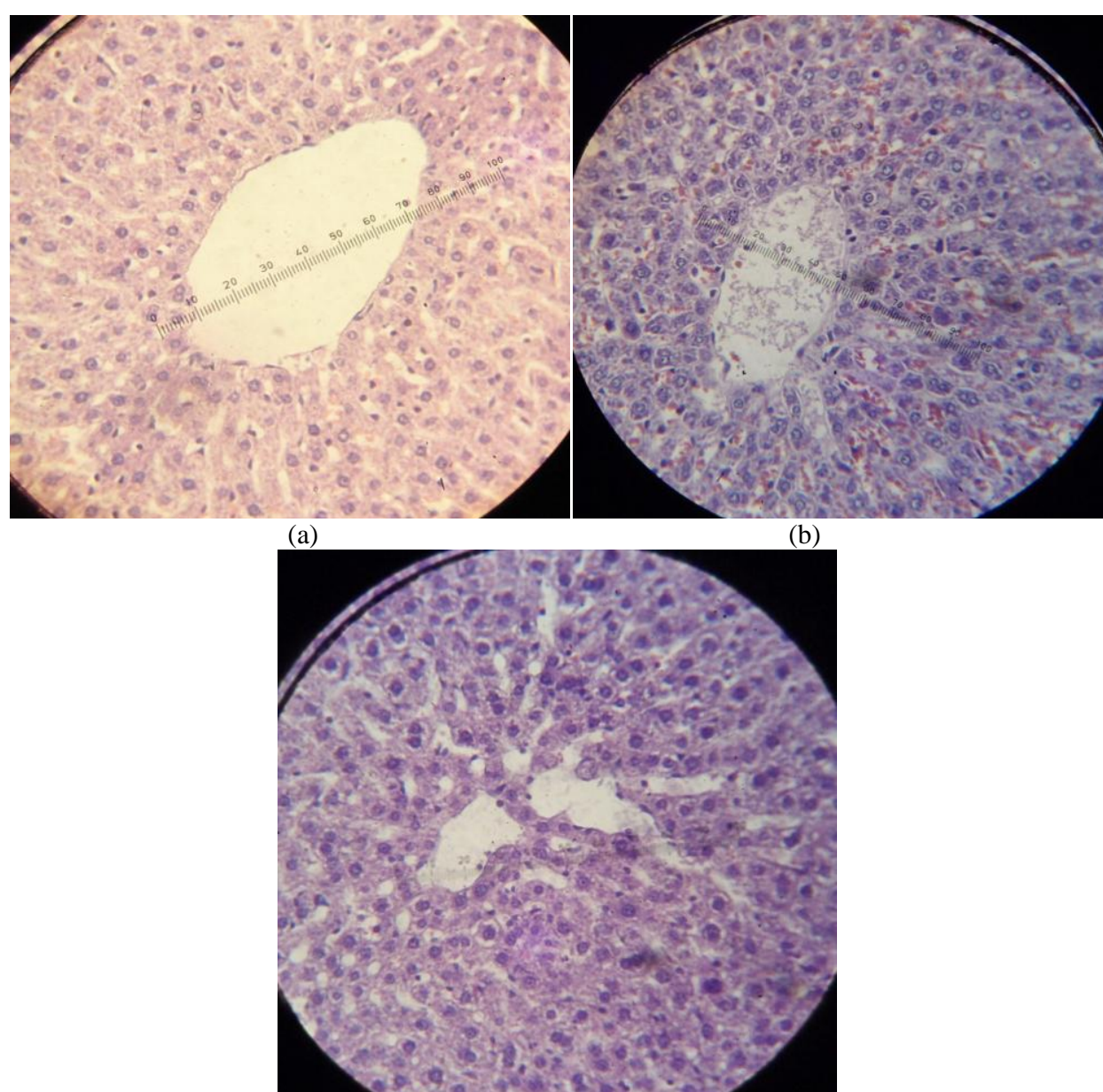

(c)

Fig. 1 Figs 1a-1c show the cross-section of liver of control (group B1 and B2), group A1 and group A2 rats respectively.

Among experimental rats given doxorubicin the liver showed disruption in histological architecture, degeneration of hepatic cords, vacuolated cells with round to oval and elliptical shaped nuclei showing apoptotic features, and dilated sinusoidal space. Pathological changes such as necrosis, irregular cell membrane, irregular clumping of chromatin like mass were also seen. In a study in the fetal rat liver by Pedrycz et al. the rats treated with doxorubicin demonstrated the apoptotic features. Perisinusoidal spaces were often dilated and swollen with the content of damaged hepatocyte cytoplasm and connective tissue proliferation was observed [10].

In another similar study done in male rats by Hassan et al. the most pronounced histological abnormalities in the rats treated with doxorubicin at a dose of $1 \mathrm{mg} / \mathrm{kg}$ body weight on alternate days for 20 days involved the dissolution of hepatic cords which appeared as empty vacuoles aligned by strands of necrotic hepatocytes. The hepatic tissues showed the presence of dense focal inflammatory cells or necrotic tissues. Higher tendency for liver fibrosis manifested by the presence of many spots of focal cellular granulomatous lesions were also the features of DOX treated rats [12]. However in the present study the dissolution of the hepatic cords were rare which might be attributed to the single dose of doxorubicin rather than the cumulative dose. Doxorubicin causes an imbalance between free oxygen radicals and antioxidants. The disturbance in oxidant-antioxidant systems results in tissue injury [13].

\section{Conclusion}

In conclusion, the present study reveals changes in 
hepatocyte size, nucleus diameter and disruption of normal architecture of liver in rats given doxorubicin. Thus it seems that doxorubicin is hepatotoxic in animals so care needs to be taken during doxorubicin chemotherapy.

\section{Acknowledgements}

We kindly acknowledge B P Koirala Institute of Health Sciences for the support in the study.

\section{References}

[1] Osama, A. H. A., and Suzan, B. S. A. 2008. "Ultrastructural Studies on the Changes Induced by Toxic Effect of Doxorubicin on Rat Hepatocyte and Protective Role of Dexrazoxane." Biosciences, Biotechnology Research Asia 5 (2): 551-7.

[2] Saalu, L. C., Enye, L. A., and Osinubi, A. A. 2009. “An Assessment of the Histomorphometric Evidences of Doxorubicin-Induced Testicular Cytotoxicity in Wistar Rats." International Journal of Medicine Sciences 1 (9): 370-4.

[3] Sadeghi-aliabdi, H., Minaiyan, M., and Dabestan, A. 2010. "Cytotoxic Evaluation of Doxorubicin in Combination with Simvastatin against Human Cancer Cells." Research in Pharmaceutical Sciences 5 (2): 127-33.

[4] Single, P. K., Deally, C. M., and Weinberg, L. E. 1987. "Subcellular Effects of Andriamycin in the Heart; A Concise Review.” J. Mol. Cardiol. 99: 817-28.

[5] Ballet, F., Vrignaud, P., Robert, J., Rey, C., and Poupon, R. 1987. "Hepatic Extraction, Metabolism and Biliary Excretion of Doxorubicin in the Isolated Perfused Rat Liver." Cancer Chemother Pharmaco. 19: 240-5.

[6] Dodion, P., Bernstein, A. L., Fox, B. M., and Bachur, N. R. 1987. "Loss of Fluorescence by Anthracycline Antibiotics:
Effects of Xanthine Oxidase and Identification of the Nonfluorescent Metabolites." Cancer Res. 47: 1036-9.

[7] Le Couteur, D. G., Fraser, R., Hilmer, S., Rivory, L. P., and McLean, A. J. 2005. "The Hepatic Sinusoid in Aging and Cirrhosis: Effects on Hepatic Substrate Disposition and Drug Clearance." Clin. Pharmacokinet 44 (2): 187-200.

[8] Manov, I., Bashenko, Y., Wolkowicz, A. E., Mizrahi, M., Liran, O., and Lancu, T. C. 2007. "High-Dose Acetaminophen Inhibits the Lethal Effect of Doxorubicin in HepG2 Cells: The Role of P-glycoprotein and Mitogen-Activated Protein Kinase p44/42 Pathway." Journal of Pharmocology and Experimental Therapeutics 322 (3): 1013-22.

[9] Murat, Y., Orhan, B., Hakan, M., Onder, S., Ahmet, N., Ozcan, K. et al. 2007. "Protective Effects of Erdosteine on Doxorubicin-induced Hepatotoxicity in Rats." Medical Research 38: 380-5.

[10] Pedrycz, A., Boratynski, Z., Wieczorski, M., and Visconti, J. 2005. "Ultrastructural and Immunohistochemical Evaluation of Apoptosis in Foetal Rat Liver after Adriamycin Administration." Bull Vet Inst. Pulway 49: 475-8.

[11] Kelvin, H., Afsheen, K., Eleni, L., Torbenson, M. S., Georgiades, C. S., and Geschwind, J. F. 2006. "New Intra-arterial Drug Delivery System for the Treatment of Liver Cancer: Preclinical Assessment in a Rabbit Model of Liver Cancer." Clin. Cancer Res. 12: 2563-7.

[12] EI-Sayyad, H. I., Ismail, M. F., Shalaby, F. M., Abou-EI-Magd, R. F., Gaur, R. L., Fernando, A. et al. 2009. "Histopathological Effects of Cisplatin, Doxorubicin and 5-flurouracil (5-FU) on the Liver of Male Albino Rats." Int. J. Biol. Sci. 5 (5): 466-73.

[13] Karaman, A., Fadillioglu, E., Turkmen, E., Tas, E., and Yilmaz, Z. 2006. "Protective Effects of Leflunomide against Ischemia Reperfusion Injury of the Rat Liver." Pediatr Surg. Int. 22: 428-34. 